\title{
Optical investigation of bovine grey and white matters in visible and near-infrared ranges
}

\author{
Ali SHAHIN ${ }^{\mathrm{a}, \mathrm{b},{ }^{*}, \text { Wesam BACHIR }}{ }^{\mathrm{a}, \mathrm{c}}$, Moustafa Sayem EL-DAHER ${ }^{\mathrm{a}, \mathrm{d}}$ \\ ${ }^{a}$ Biomedical Photonics Laboratory, Higher Institute for Laser Research and Applications, Damascus University, Damascus, \\ Syria \\ ${ }^{b}$ Physiology Department, Veterinary Faculty, Hama University, Hama, Syria \\ ${ }^{c}$ Faculty of Informatics Engineering, Al-Sham Private University, Damascus, Syria \\ ${ }^{d}$ Arab International University, Damascus, Syria \\ *E-mail address: ali.shahin88@yahoo.com
}

\begin{abstract}
Introduction: Due to enormous interests for laser in medicine and biology, optical properties characterization of different tissue have be affecting in development processes. In addition, the optical properties of biological tissues could be influenced by storage methods. Thus, optical properties of bovine white and grey tissues preserved by formalin have been characterized over a wide wavelength spectrum varied between $440 \mathrm{~nm}$ and $1000 \mathrm{~nm}$.

Materials and Methods: To that end, a single integrating sphere system was assembled for spectroscopic characterization and an inverse adding-doubling algorithm was used to retrieve optical coefficients, i.e. reduced scattering and absorption coefficients.

Results: White matter has shown a strong scattering property in comparison to grey matter. On the other hand, the grey matter has absorbed light extensively. In comparison, the reduced scattering profile for both tissue types turned out to be consistent with prior works that characterized optical coefficients in vivo. On the contrary, absorption coefficient behavior has a different feature.

Conclusion: Formalin could change the tissue's optical properties because of the alteration of tissue's structure and components. The absence of hemoglobin that seeps out due to the use of a formalin could reduce the absorption coefficient over the visible range. Both the water replacement by formalin could reduce the refractive index of a stored tissue and the absence of hemoglobin that scatters light over the presented wavelength range should diminish the reduced scattering coefficients over that wavelength range.
\end{abstract}

Key words: white matter; grey matter; reduced scattering, absorption; reduced albedo.

\section{Introduction}

Biological tissue optical properties are regarded as a basic requirement for providing an insight into light-tissue interaction. The development of optical diagnostic and therapeutic methods highly depends on tissue optical characteristics. However, the light energy distribution is the basic challenge that relates to tissue optical parameters and plays a vital role in dosimetry and therapy. ${ }^{1-4}$ Tissue optical coefficients have associated with tissue structure and type. Furthermore, the scattering coefficient provides information about scattering component form and concentration, whereas the absorption coefficient can lead to chromophore type and concentration. Thus, optical parameters might be utilized to identify and localize unhealthy tissues ${ }^{5-10}$ Yaroslavsky et al. investigated the optical characteristics of native and coagulated human brain tissue over ultraviolet to the near infrared region using a double integrating sphere and inverse Monte Carlo
(IMC). ${ }^{11}$ The absorption coefficient of all specimens turned out to be small in comparison to their scattering coefficient and the highest penetration depth was found in the wavelength range between $1000 \mathrm{~nm}$ and $1100 \mathrm{~nm}$. The study also found that thermal coagulation decreases the effective penetration depth of all tissue types ${ }^{11} \mathrm{~A}$ work by Gebhart et al. retrieved brain optical properties based on inverse adding doubling (IAD). ${ }^{12}$ Discrepancies of their findings can be explained by several factors such as the corresponding approach implemented to analyze the spectral characteristics and sample preparation method. ${ }^{11,12}$ Optical properties of lamb brain tissue were investigated over the visible and near-infrared regions for native and coagulated samples. The differences between the optical properties of the lamb and the human brain were discussed. ${ }^{13}$ Azimipour et al. extracted in vitro the rat brain optical properties using three different cuts that have a $500 \mu \mathrm{m}$ thickness at three different wavelengths (405, 532 and

(C) 2021 Ali Shahin, Wesam Bachir, Moustafa Sayem El-Daher. This is an open access article licensed under the Creative Commons Attribution-NonCommercialNoDerivs License (http://creativecommons.org/licenses/by-nc-nd/4.0/). 
$635 \mathrm{~nm}) .{ }^{14}$ Reduced scattering coefficient turned out to be high at the brain center and gradually reduced to the surface, as opposed to the absorption coefficient. The absorption coefficient varied from 0.1 to $0.35 \mathrm{~mm}^{-1}$ and between 1.5 and $5 \mathrm{~mm}^{-1}$ for the reduced scattering coefficient at $532 \mathrm{~nm} .{ }^{14}$ On the other hand, preparation and storage methods of a tissue sample and their influences on tissue optical coefficients have been investigated. Formalin fixation is a preparation method widely used for the long-time storage of tissue specimens. Major prior works aimed to study formalin fixation influence, predicted an enormous deviation of fixed tissue optical properties. However, formaldehyde acts as a cross-linking agent at the molecular level for soluble and structural proteins, which could cause a change of scattering property whereas absorption remains unaltered. ${ }^{15-17}$ In contrast, OCT image for formalin-fixed tissue shown the absence of blood vessels following storage that has predicted a decreasing of absorption spectra. ${ }^{18}$ For more understanding, Pitzschke et al. investigated the reduced scattering, absorption and effective attenuation coefficient of rabbit brain tissue at 635,671 and $808 \mathrm{~nm}$ to understand the effects of formalin storage on their optical properties in comparison to in vivo measurements. ${ }^{19}$ Although, tissue-stored absorption and reduced scattering coefficients increased at every wavelengths in comparison to in vivo results, absorption and reduced scattering profiles have had a decremental relationship with increased wavelength over the studied range to be similar the behaviour of in vivo-measured optical parameters with wavelength. ${ }^{19}$ In other words, using a formalin in order to store brain tissues resulted in a quantitative disagreement of the measured optical characteristics, while no qualitative variations could be observed. ${ }^{19}$ On the other hand, Anand et al. observed a dissimilarity of the formalin-fixed tissue's absorption spectrum in comparison to freshly sample, which has been explained by seeping out of hemoglobin from red cells. ${ }^{20}$ Furthermore, reduced scattering coefficient has remained a constant value for formalin fixed samples and an exponential decrease with wavelength has been estimated for fresh sample. ${ }^{20}$ Thus, there were qualitative and quantitative variations caused by using a formalin for storing tissues that turned out to be inconsistent with another work. ${ }^{19,20}$ Finally, the consequences of using the formalin for brain tissue preservation and their influence on the optical properties have not been decided yet and with the best of our knowledge, the changes of utilizing that method of storing on the optical properties of white and grey matters have not been studied.

In order to determine the optical properties of biological tissue, numerical solutions of the radiative transfer equation (RTE) are regarded as the best choice to be used with indirect techniques. These depend on the radiometric characteristics to reconstruct the optical coefficients of the present matter. ${ }^{21-23}$ Inverse adding doubling (IAD) algorithm needs three raw intensities to be used, diffuse reflectance $R_{d}$, total transmittance $\mathrm{T}$, and collimated transmittance $\mathrm{T}_{\mathrm{c}}$. IAD algorithm repeatedly solves radiative transfer equation (RTE) until the calculated values match the measured radiometric parameters. ${ }^{22-24}$ This method could be useful for any material types without taking into account albedo and the ratio between scattering and absorption unlike the approximation solution that could be applied with some presumptions. ${ }^{22-25}$ Beside inverse Monte Carlo method, inverse adding-doubling algorithm was mainly used to investigate optical parameters by several prior research groups and their precision has been recognized. ${ }^{10-14}$

The aim of this work is to characterize the optical properties of grey and white matters of bovine brain, fixed with formalin. In order to determine the reduced scattering and absorption coefficients, a single integrating sphere system was used. Spectral measurements were utilized to predict absorption and reduced scattering coefficients using inverse adding doubling algorithm (IAD). ${ }^{23-26}$ Consequently, the presented findings have been discussed and compared with prior works.

\section{Materials and Methods}

\section{Sample Preparation}

Firstly, this section of the experiment was approved by the ethical committee at Damascus University, which was conducted according to established animal welfare guidelines and compiles with all regulations. Then, two different types of bovine brain tissue were prepared for optical investigation. Shortly, after killing, a bovine brain was stored in formalin. Then, the specimens were sectioned as follows: seven samples of grey matter (cerebral cortex) with similar width, height and thickness $(1.5 \times 1.5 \times 0.1 \mathrm{~cm})$ were created and five samples of white matter were sectioned to have $1.2 \mathrm{~mm}$ thickness and same width and height of grey sections. All samples were sandwiched between two glass slides that have a $1 \mathrm{~mm}$ thickness each. Refractive index of glass slides and tissue samples were approximated to 1.57 and 1.4 respectively. ${ }^{12}$

\section{Experimental Setup}

In order to retrieve absorption and reduced scattering coefficients of bovine brain tissue using inverse addingdoubling algorithm, single integrating sphere (819C-IS-5.3, Newport, U.S.A) was used. Diffuse reflectance Rd and total transmittance $\mathrm{T}$ measurements applied in the same setting, as shown in Figure 1. Broadband beam emanated from a halogentungsten light source (HL-2000-HP-FHSA, Ocean Optics, U.S.A) was focused to have a $4 \mathrm{~mm}$ diameter on a sample of interest through a convex lens (focal length, $f=10 \mathrm{~cm}$ ). Tissue sample was sandwiched between two slides of glass and hold by a special holder belongs that integrating sphere. Then, emanated light was delivered using an optical fiber (diameter, $\phi=400 \mu \mathrm{m}$ ) to a portable spectrometer (USB4000, Ocean Optics, U.S.A) that is connected to a PC through a USB interface. 
(a)

Diffuse Reflectance Measurement

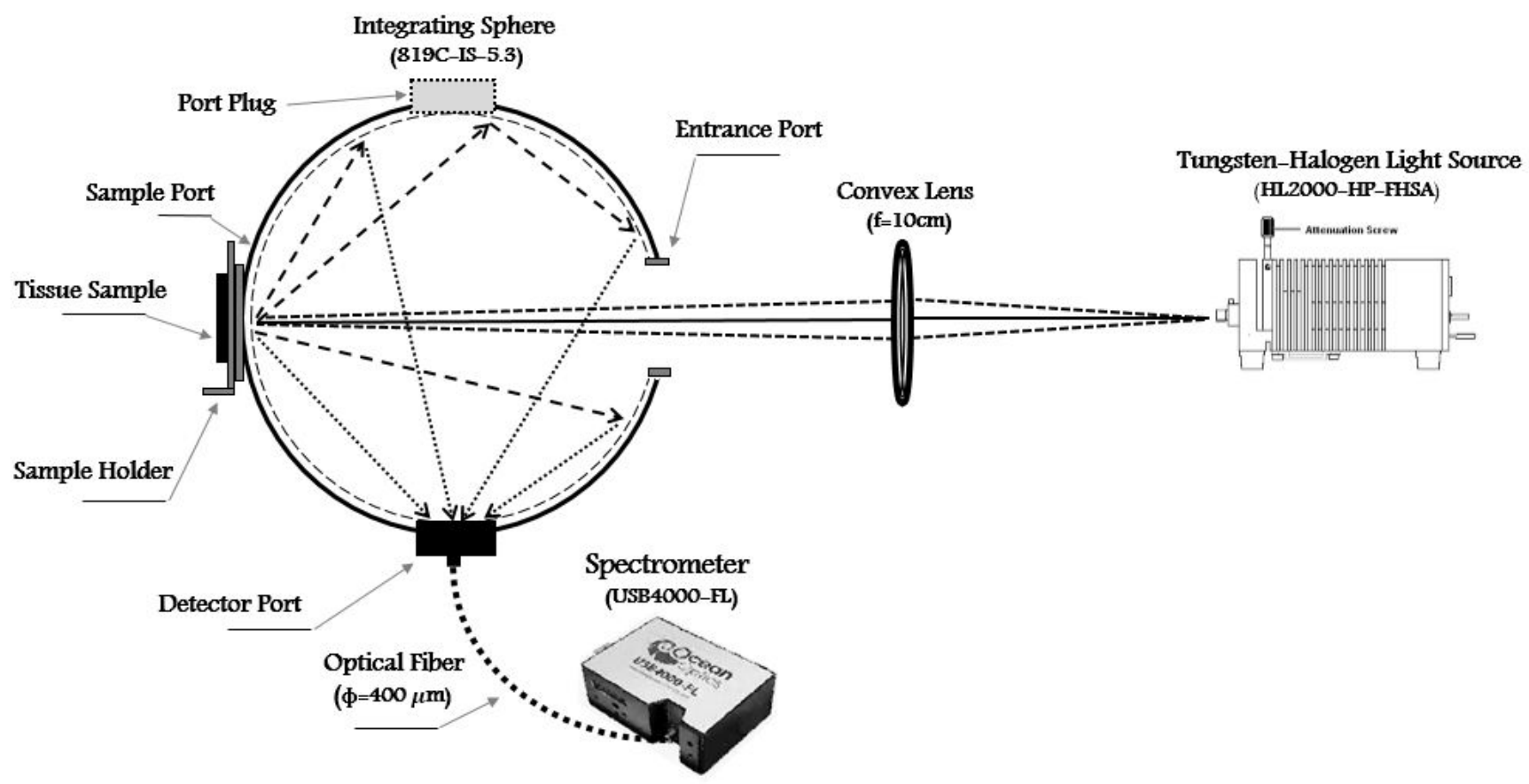

(b)

Total Transmittance Measurement

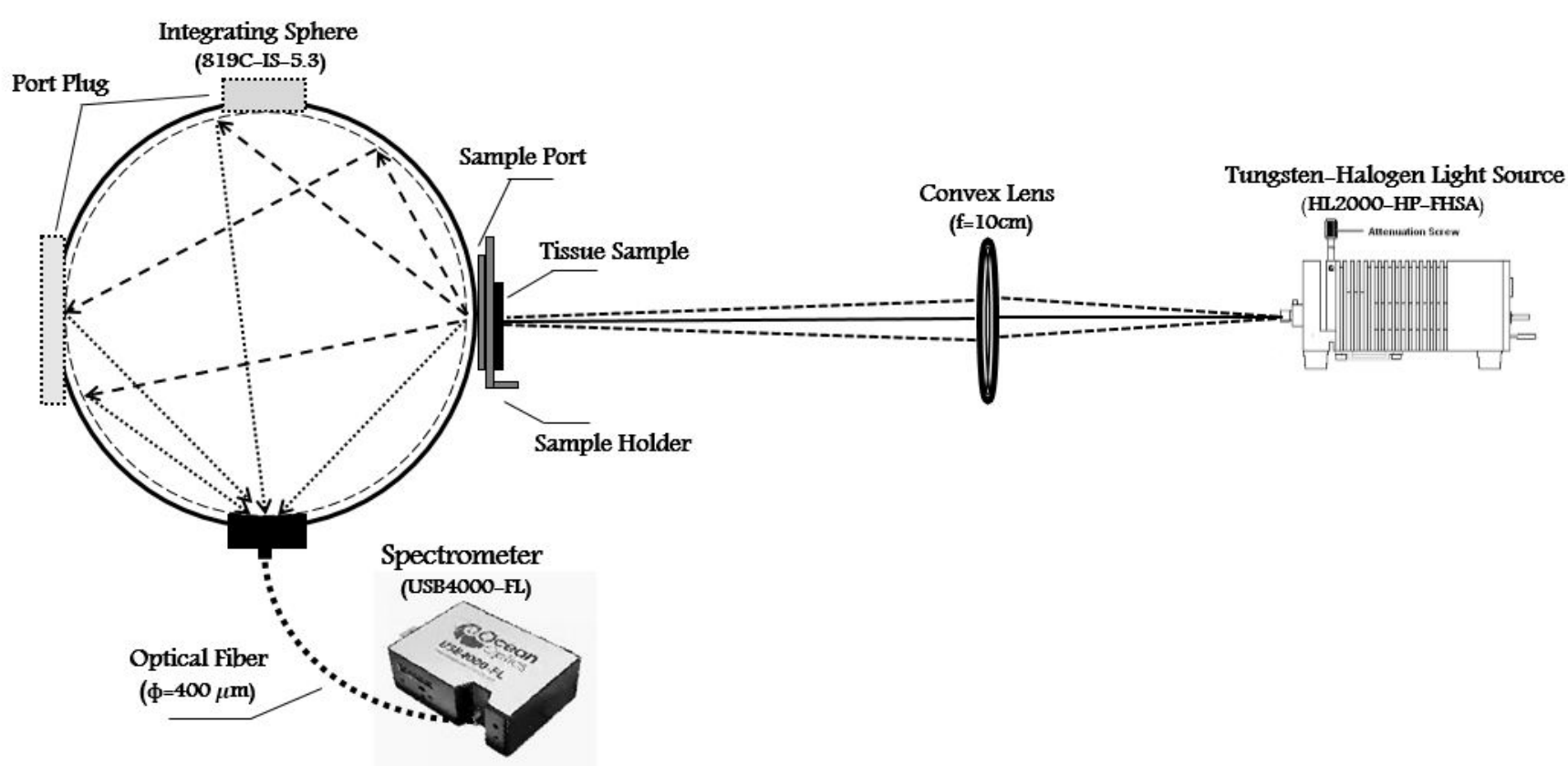

Figure 1. A single integrating sphere system. (a) Schematic drawing represents a diffuse reflectance (Rd) measurement setup, tungstenhalogen light source was utilized to generate a focused beam using a convex lens (focal length, $\mathrm{f}=10 \mathrm{~cm}$ ). Then, tissue illuminated via $4 \mathrm{~mm}$ spot light. and diffused light collected via an optical fiber (diameter, $\phi=400 \mu \mathrm{m}$ ) that connected to spectrometer. (b) Schematic drawing of total transmittance (T) measurement setup, tungsten-halogen light source was used to generate a focused beam using a convex lens (focal length, $f=10 \mathrm{~cm}$ ). Then, tissue sample was illuminated via $4 \mathrm{~mm}$ spot light diameter. Diffused light collected via an optical fiber (diameter, $\phi=400 \mu \mathrm{m})$ that connected to a spectrometer. 


\section{Data Processing Technique}

For data processing and optical properties estimation, inverse adding-doubling code has been used that correspondence to Windows XP, which developed by Scott Prahl and supplied with Monte Carlo calculation to take into account the light lost out the edges of samples. ${ }^{23,26}$ However, the above-mentioned algorithm required some experimental data belongs the integrating sphere used and measuring total transmittance and diffuse reflectance in order to retrieve absorption and reduced scattering coefficients. Finally, we can summarized the experimental in-puts regarding IAD code as follows:

1. Light beam diameter $(\phi=4 \mathrm{~mm})$.

2. Sample thickness $\left.\left(\mathrm{d}_{\text {grey }}=1 \mathrm{~mm}\right) \& \mathrm{~d}_{\text {white }}=1.2 \mathrm{~mm}\right)$.

3. Glass slide thickness $\left(\mathrm{d}_{\text {glass slides }}=1 \mathrm{~mm}\right)$.

4. Refractive indexes of glass slide $\left(n_{\text {glass }}=1.57\right)$ and tissue sample $\left(\mathrm{n}_{\text {brain tissue }}=1.4\right)$.

5. Integrating sphere diameter $(\phi=134.6 \mathrm{~mm})$.

6. Diameters for sample port $(\phi=25.4 \mathrm{~mm})$, detector port $(\phi=$ $400 \mu \mathrm{m})$ that matches fiber core diameter.

7. Reflectivity of the inner wall was approximated to 0.97 .

\section{Results}

Based on a single integrating sphere system, total transmittance and diffuse reflectance could be measured. Figure 2 presents mean values of total transmittance and diffuse reflectance of grey samples. As can be shown in Figure 2a, total transmittance progressively increased with increased wavelength. Diffuse reflectance is directly proportional to increased wavelength over the sub-spectrum ranged between $440 \mathrm{~nm}$ and $600 \mathrm{~nm}$. Then, that turned out to be stable over the rest spectrum, Figure 2b. Regarding white tissue, spectral characteristics of all samples were measured and their averaged values are presented in Figure 3. As can be seen in Figure 3a, total transmittance of white matter is directly proportional to increased wavelength. Although the white matter sample has a thicker section, it has a valuable transmittance in comparison to grey matter. Furthermore, white matter has exhibited high reflection in comparison to grey matter, Figure $\mathbf{3 b}$.

Optical coefficients of all grey tissue samples were predicted depending on spectral characteristics and the mean values were determined in relation to wavelength. Figure $4 \mathbf{a}$ presents average values of reduced scattering coefficient that decreased progressively with increased wavelength over the present wavelength range. Its values varied between $0.406 \pm 0.0457$ $\mathrm{mm}^{-1}$ at $441 \mathrm{~nm}$ and $0.2072 \pm 0.0122 \mathrm{~mm}^{-1}$ at $996 \mathrm{~nm}$. Figure 4b depicts the relationship between absorption coefficient and wavelength that found to be inversely proportional to increased wavelength. Accordingly, remarkable changes could be observed with shorter wavelengths and absorption property varied between $0.196 \pm 0.0285 \mathrm{~mm}^{-1}$ at $441 \mathrm{~nm}$ and $0.0377 \pm 0.0052 \mathrm{~mm}^{-1}$ at $600 \mathrm{~nm}$. Insignificant decline in absorption feature may be seen over the remaining wavelength spectrum ranged from $600 \mathrm{~nm}$ to $1000 \mathrm{~nm}$. The relationship between grey matter optical coefficients and wavelength could be estimated by fitting curve procedure in Matlab (Mathworks 2014, U.S.A) and given by:

$\mu_{a}=3.447 * 10^{13} * \lambda^{-5,423}+0.01217$

$\mathrm{Eq} \cdot 1$

$\mu_{s}^{\prime}=65.69 * \lambda^{-0.846}$

Accordingly, two critical optical parameters could be evaluated depending on the previous investigations, optical penetration depth and reduced albedo, Figure 4. Figure 4c presents the relationship between grey tissue penetration depth and wavelength that estimated via absorption and reduced scattering coefficient of grey matter. However, the optical penetration depth turned out to be higher with longer wavelength despite of unsystematic changes over wavelength.

For white matter optical characterization, reduced scattering and absorption coefficients were predicted for all white matter samples and mean values were calculated. Figure 5a depicts the relation between reduced scattering coefficient and wavelength of white matter. Approximately, reduced scattering is inversely proportional to increased wavelength over the present wavelength range. Its values varied from $0.419 \pm$ $0.0224 \mathrm{~mm}^{-1}$ at $441 \mathrm{~nm}$ and $0.239 \pm 0.029 \mathrm{~mm}^{-1}$ at $996 \mathrm{~nm}$. As a result, noticeable changes could be approximated to a power relation using fitting curve function, Equation 2. On the other hand, absorption coefficient turned out to be decreased with increased wavelength and the lowest values could be estimated in the near infrared region (therapeutic window), Figure 5b. Its values ranged from $0.0825 \pm 0.0140 \mathrm{~mm}^{-1}$ at $441 \mathrm{~nm}$ to $0.00327 \pm 0.00228 \mathrm{~mm}^{-1}$ at $996 \mathrm{~nm}$. The fitting curve procedure may be utilized to predict the equation combining white matter's optical coefficients and wavelength and expressed by:

$\mu_{a}=1.493 * 10^{5} \cdot \lambda^{-2.319}-0.01676$

Eq. 2

$\mu_{s}^{\prime}=17.6 * . \lambda^{-0.5924}$

Figure 5c presents optical penetration depth for white matter that turned out to be much larger for longer wavelengths. Depending on absorption and reduced scattering coefficients, reduced albedo for white matter could be estimated, Figure 5d. 
(a)

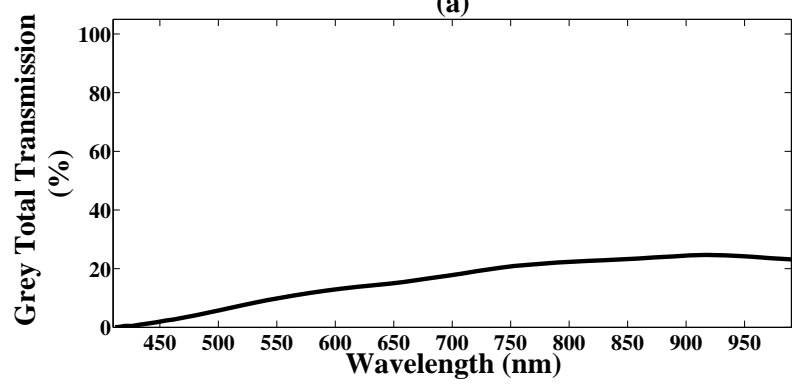

(b)

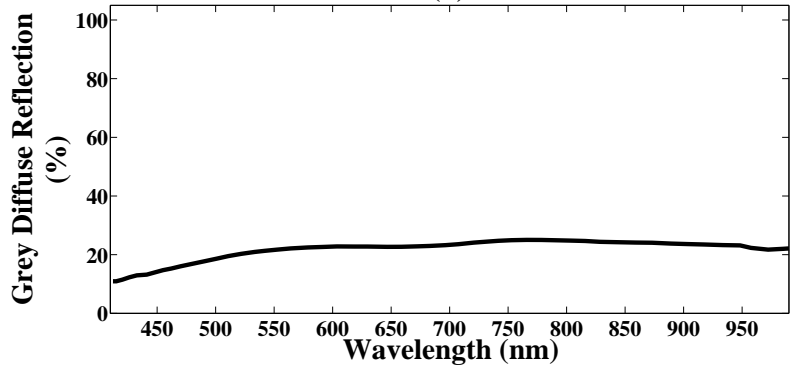

Figure 2. Spectral characteristics of grey matter. (a) Correlation of average values for total transmittance of bovine grey matter with wavelength over visible and near infrared ranges, (b) Relationship between averaged values of diffuse reflectance with wavelength for bovine grey matter over visible and near infrared ranges.

(a)

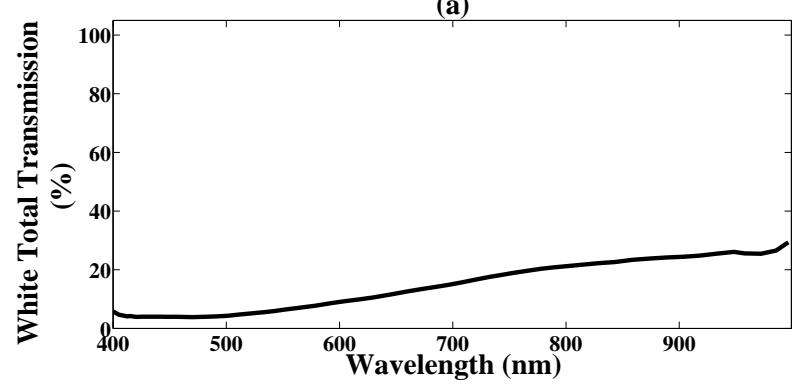

(b)

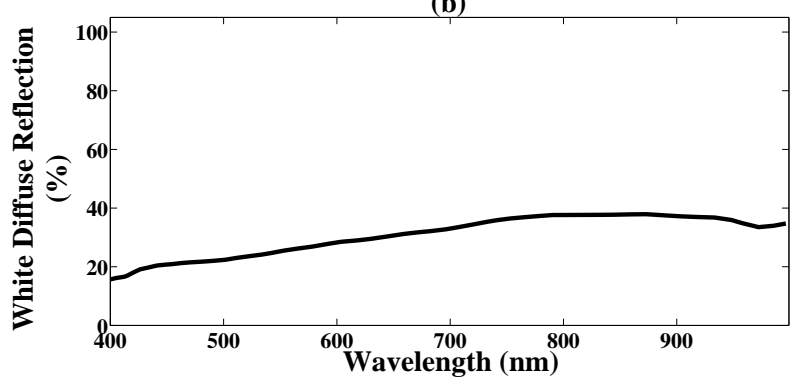

Figure 3. Spectral characteristics of white matter. (a) Correlation of average values of total transmittance for bovine white matter and wavelength over visible and near infrared ranges, (b) Correlation of average values of diffuse reflectance for bovine white matter and wavelength over visible and near infrared ranges.

(a)

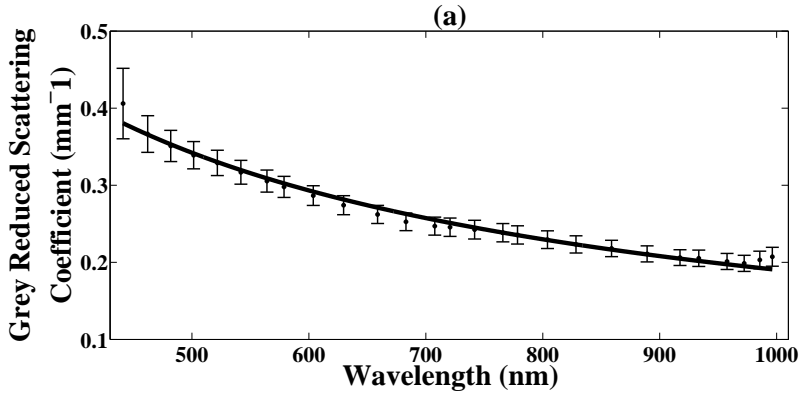

(c)

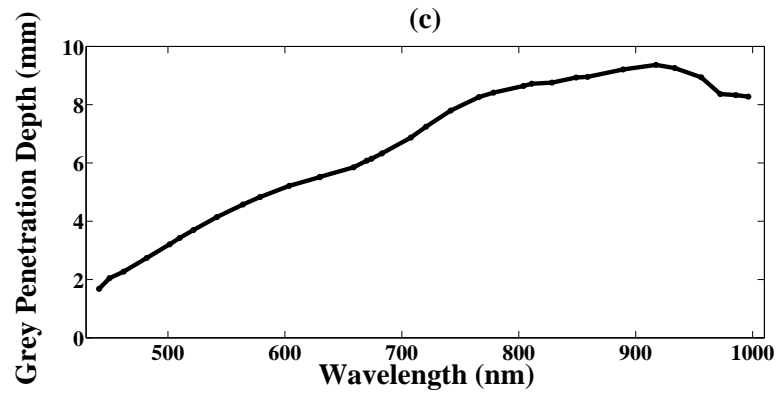

(b)

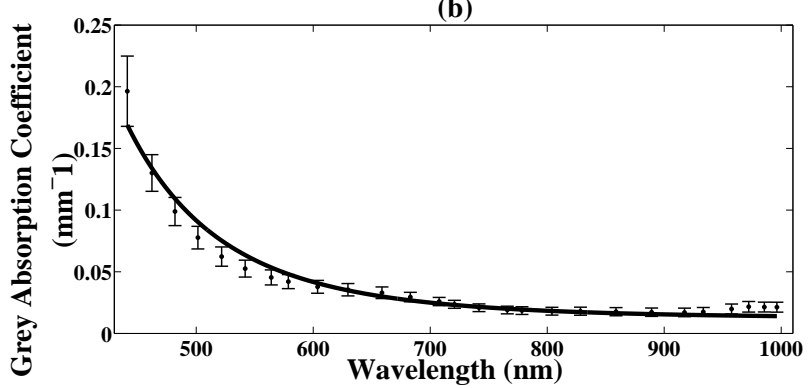

(d)

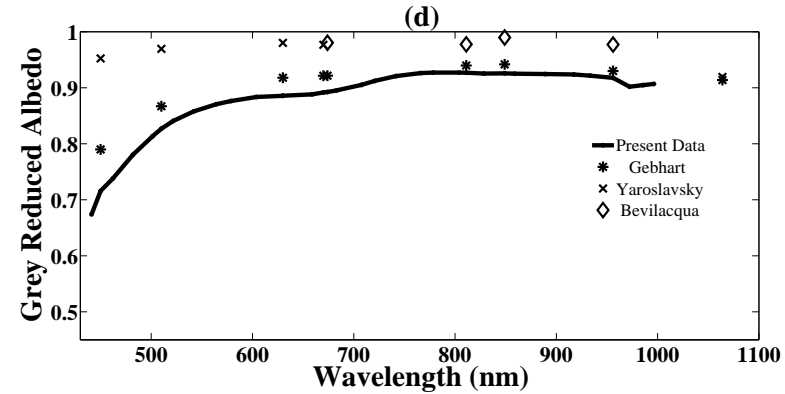

Figure 4. Optical properties of grey matter. (a) Correlation of average values of reduced scattering coefficient for grey matter with wavelength over visible and near infrared ranges. (b) Relationship between averaged values of absorption coefficient for grey matter and wavelength over visible and near infrared ranges. The error bar represents the standard deviation for 7 samples of grey matter. (c) Variation of grey matter optical penetration depth with wavelength over visible and near infrared ranges. (d) Correlation of grey matter reduced albedo and wavelength besides to results predicted by prior works. 

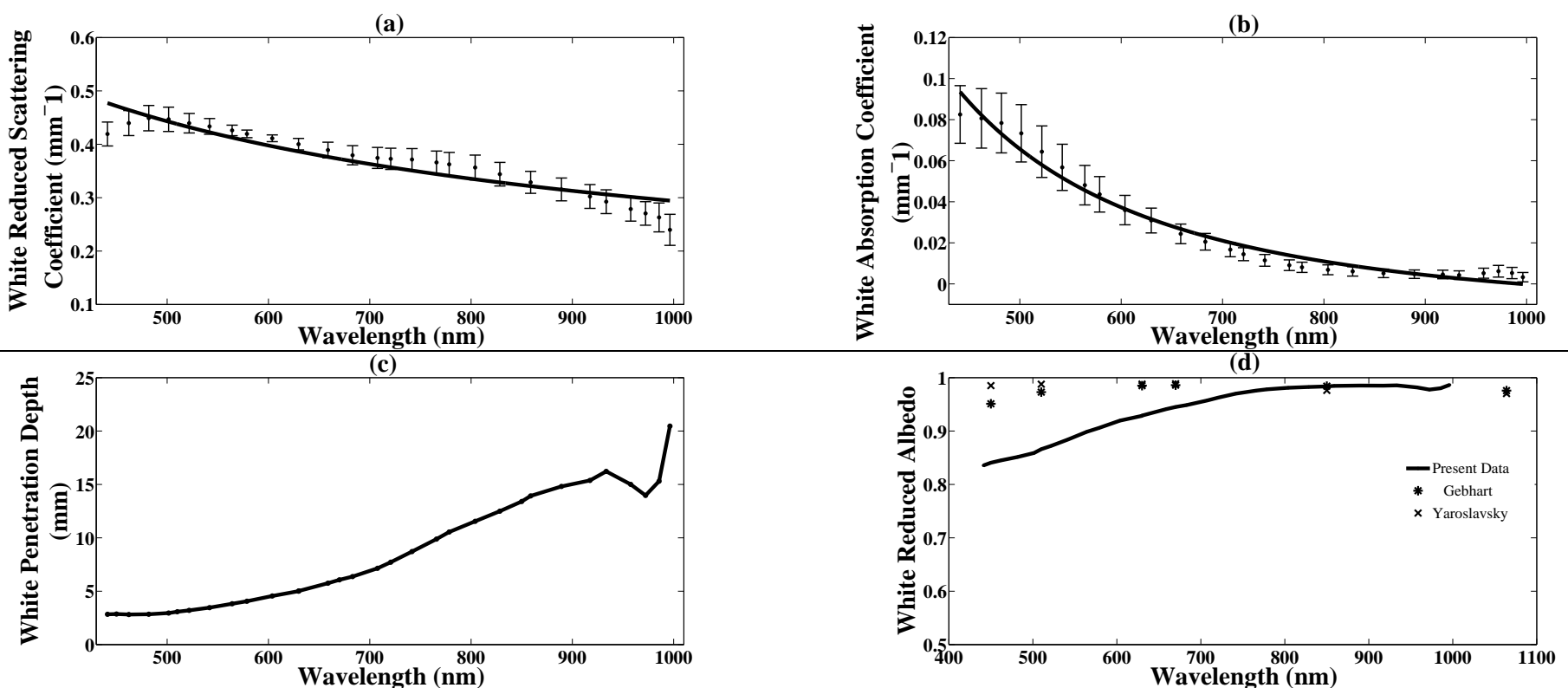

Figure 5. Optical properties of white matter. (a) Relationship between averaged values of reduced scattering coefficient for white matter and wavelength over visible and near infrared ranges. (b) Relationship between averaged values of absorption coefficient for white matter and wavelength over visible and near infrared ranges. The error bars represents the standard deviation for 5 samples of white matter. (c) Variation of white matter optical penetration depth with wavelength over visible and near infrared ranges. (d) Relationship between white matter reduced albedo and wavelength besides to results predicted by prior works.

\section{Discussion}

Since bovine white and grey matters that fixed by formalin have been studied in the present work besides to that the major previous works aimed to characterize optical properties of white and grey matter have used fresh human tissues without adding formalin. Thus, the quantitative comparison of absorption and reduced scattering coefficients could not be applied and the comparison has been limited to be qualitative by comparing the behaviour of optical coefficients' variations with wavelength to previous works. Indirect method was utilized to characterize tissue sample optical properties depending on a single integrating sphere system and inverse adding doubling algorithm (IAD). Figure 4 depicts the grey matter's reduced scattering and absorption coefficients and their correlation with wavelength. Then, because of the varying dimensions for subcellular organelles, tissue scattering feature lies between Rayleigh and Mie regimes. ${ }^{10-13}$ However, grey reduced scattering intensity showed that it is inversely proportional to increased wavelength and comparable to a power equation, Equation 1, the exponent of the inverse power relationship lied between Mie and Rayleigh limitations that corresponds to the previous expectations that estimated from in vivo measurements. ${ }^{11-13,27}$ Then, Pitzschke et al approved that the reduced scattering profile for formalin-fixed tissue decreased with wavelength increases over the wavelength range varied between $635 \mathrm{~nm}$ and $808 \mathrm{~nm}$, which turned out to be correspondence to our findings and inconsistent with Anand results. ${ }^{19,20}$ On the other hand, absorption coefficient variation is inversely proportional to wavelength and decreased dramatically in the sub-range from $441 \mathrm{~nm}$ to $600 \mathrm{~nm}$, after that the absorption have showed to be more stable for larger wavelengths. The absorption coefficient profile turned out to be inconsistent with fresh tissue's absorption profile and agreed with the studies implemented on formalin-fixed tissues. ${ }^{11-13,19,20}$ The existence of melanin and water as the main absorbers reveals the degradation of grey matter absorption variation with increased wavelength over the present range. Because of using a formalin fixation method for tissue storage, hemoglobin absorption peaks could not be distinguished and tissue absorption feature may resemble melanin and water characteristics. ${ }^{18-20}$ Depending on grey absorption and reduced scattering coefficients, reduced albedo might be extracted that varied from 0.673 at $441 \mathrm{~nm}$ to 0.906 at $996 \mathrm{~nm}$. That directly increases with wavelength over a spectrum ranged between $441 \mathrm{~nm}$ and $500 \mathrm{~nm}$, and then a stable relationship could be seen for higher wavelengths. In comparison, the present results of grey matter turned out to be in agreement with Gebhart findings despite the subtle differences and the systematic errors that varied between $9.3 \%$ at $450 \mathrm{~nm}$ and $1.3 \%$ at $956 \mathrm{~nm} .^{12}$ Furthermore, Yaroslavsky prediction was higher and errors varied between $24.8 \%$ at $450 \mathrm{~nm}$ and $8.7 \%$ at $670 \mathrm{~nm}$ and in comparison with Bevilacqua findings the error varied between $8.9 \%$ and $6.9 \%$ as can be seen in Figure $4 d^{10,-12}$ Regarding white matter's optical characteristics, reduced scattering coefficient shows inverse relationship to increased wavelength. That might be approximated to a power equation to simulate a case between Rayleigh and Mie scatterings, Equation 2, which turned out to be consistent with previous findings extracted by measurements on fresh white brain tissue. ${ }^{11,12}$ However, reduced scattering behaviour has agreed with findings extracted from Pitzschke and has not agreed with Anand results, which revealed that the reduced scattering remained unaltered with wavelength increases over the visible range. ${ }^{19,20}$ However, white matter absorption may be featured by melanin, 
and water contributions and thus the absence of blood should enormously affect its values, that explained the differences between the present results and prior works applied in vivo. ${ }^{11,12}$ Figure 5d represents the correlation of white matter reduced albedo and wavelength that slightly increased with increased wavelength to be varied between 0.83 at $441 \mathrm{~nm}$ and 0.98 at $996 \mathrm{~nm}$. The respective measurements were matched to prior works regarding fresh white matter over the sub-spectrum from $750 \mathrm{~nm}$ to $1000 \mathrm{~nm}^{11,12}$ The overestimation could be noticed with shorter wavelengths and systematic errors turned out to be not more than $11 \%$ for Gebhart findings and $14 \%$ for Yaroslavsky results. ${ }^{11,12}$ Although the existence of quantitative differences in optical characteristics between two kinds of brain tissue, optical coefficients profile found to be in accord. Accordingly, grey matter may be regarded as a highly absorbing medium than white matter which has further scattered the incident beam. ${ }^{11,12}$ White tissue is marked by a melanin leakage in comparison to grey matter as well as the presence of fat in white matter. ${ }^{4,22}$ Fat tissue contributes to scattering property and absorption around $1000 \mathrm{~nm}$, which could explain a relative slight increase in absorption coefficient at the end of the studied spectrum. Low concentration of melanin at white matter may be regarded as the main reason for reducing absorption in comparison to grey matter. ${ }^{4}$

Obviously, the reduced scattering behavior for both white and grey tissues was in accordance with prior works, although there were quantitative reductions in comparison to fresh tissues. ${ }^{10-12}$ Regarding absorption feature, dissimilar behaviour and quantitative disagreement might be predicted. Overall, the existence of quantitative and qualitative dissimilarities may be resulted from some practical and physiological considerations. As mentioned above, using a formalin fixation may be responsible for the leakage of hemoglobin, which resulted in the absence of hemoglobin absorption peaks below $600 \mathrm{~nm} .^{18}$, ${ }^{28-30}$ Moreover, long time storage could cause a lowering of tissue water content, which may be regarded as an explanation of decreasing absorption coefficient over longer wavelengths. ${ }^{31}$ Therefore, absorption property of formalin-fixed tissue samples may resemble melanin and water unlike absorption coefficient estimated from measurement applied on fresh tissues. ${ }^{10-12}$ Furthermore, these could explain the degradation of absorption for white and grey matters, as shown in Figures $\mathbf{4 b}$ and $\mathbf{5 b}$. Regarding reduced scattering property, formalin can cause a tissue dehydration besides to alteration of tissue structure, these can lead to alteration of scattering coefficient, anisotropy factor and refractive index. ${ }^{17,19,31}$ Prior work that aimed to study the refractive index modification by formalin, indicated to slight degradation of fixed tissue refractive index until that to be equal to formalin refractive index due to the replacement of tissue water content by formalin. Since the refractive index of formalin is about 1.375 and 1.4 for fresh tissue, dehydration of formalin-fixed tissue can diminish the reduced scattering coefficient. ${ }^{17,31}$ Furthermore, the absence of hemoglobin can be considered as an enormous factor clarifying the degradation of the presented reduced scattering coefficient over the entire spectral range. ${ }^{29}$ Taking all the above into consideration, the decline of reduced scattering coefficient can be approved over all wavelength spectrum for formalin fixation method. Regarding the reduced scattering coefficient trend, prior work predicted both quantitative and qualitative discrepancies and reduced scattering profile maintained a stable value over the visible range to be about $1.5 \mathrm{~mm}^{-1}$ unlike fresh tissue. ${ }^{20}$ Accordingly, formalin fixation method could change unsystematically the reduced scattering coefficient profile. As mentioned above, the subtle difference in refractive index should cause a degradation of reduced scattering profile over the entire presented spectrum and the leakage of hemoglobin could diminish that coefficient. ${ }^{28-31}$ Thus, a considerable degradation of reduced scattering coefficients over the entire spectrum could be predicted. All these points refer that the reduced scattering coefficient could relatively maintain a similar profile of fresh tissue unlike prior work that predicted an enormous alteration of reduced scattering profile. ${ }^{20}$ The qualitative and quantitative convergence of the reduced albedo with previous studies considered as a good evidence for that expectation. On the other hand, using a focused beam with an inverse adding doubling algorithm (IAD) is conducive to experimental errors and using a tissue sample with small dimensions in comparison to sample port of integrating sphere quantitatively affects the optical characterization of tissue coefficients.

\section{Conclusions}

In summary, reduced scattering and absorption coefficients for bovine white and grey matters that stored in formalin have been investigated. Reduced scattering profile shows an exponential decrease over a wavelength range such as prior investigations extracted from in vivo measurements. Quantitative degradation could be produced by a formalin fixation that might cause a hemoglobin to be absent besides a tissue dehydration. These might decrease overall reduced scattering coefficient over the presented spectrum, but the scattering profile was expected to remain so, unlike the previous work. That prior work predicted the use of a formaldehyde could change both a reduced scattering profile and its values. On the other hand, the absence of hemoglobin has a marked influence on absorption coefficient. That may be verified by the absence of noticeable hemoglobin absorption peaks and considerable resemblance to melanin and water absorption trends. Briefly, formalin fixation could cause a considerable change of absorption coefficient profile rather than reduced scattering and reduce the optical coefficients in comparison to those of fresh tissues. Apparently, seeking a reference values for optical properties of tissue is of paramount importance to deepen our understanding of light-tissue interaction, which in turn plays a critical role in laser applications in medicine and biology. 


\section{Acknowledgements}

The authors thank all staff of biomedical photonics laboratory, Higher Institute for Laser Research and Applications, Damascus University for their help.

\section{References}

1. Eggert H R, Blazek V. Optical properties of human brain tissue, meninges and brain tumors in the spectral range of 200 to $900 \mathrm{~nm}$. Neurosurgery. 1987;21 (4):459-464. https://doi.org/10.1227/00006123-198710000-00003

2. Taddeucci A, Martelli F, Barilli M, Ferrari M, Zaccanti G. Optical properties of brain tissue. J Biomed Opt. 1996;1(1):117-123. https://doi.org/10.1117/12.227816

3. Sandell J, Zhu T. A review of in-vivo optical properties of human tissues and its impact on PDT. J Biophotonics. 2011;4(11):773787. https://doi.org/10.1002/jbio.201100062

4. Jacques SL. Optical properties of biological tissues: a review. Phys Med Biol. 2013;58:R37-R61. https://doi.org/10.1088/00319155/58/11/R37

5. Holmer C, Lehmann K, Wanken J, et al. Optical properties of adenocarcinoma and squamous cell carcinoma of the gastroesophageal junction. J Biomed Opt. 2007;12(1):014025-1-8. https://doi.org/10.1117/1.2564793

6. Lin WC, Toms SA, Johnson M, Jansen ED, Mahadevan-Jansen A. In vivo brain tumor demarcation using optical spectroscopy. J Photochem Photobiol. 2001;73:396-402. https://doi.org/10.1562/0031-8655(2001)0730396IVBTDU2.0.CO2

7. Mourant JR, Freyer JR, Hielscher AH, Eick AA, Shen D, Johnson TM. Mechanisms of light scattering from biological cells relevant to noninvasive optical-tissue diagnostics. Appl Opt. 1998;37:3586-3593. https://doi.org/10.1364/ao.37.003586

8. Salomatina EV, Jiang B, Novak J, Yaroslavsky A. Optical properties of normal and cancerous human skin in the visible and nearinfrared spectral range. J Biomed Opt. 2006;11(6):064026-1-9. https://doi.org/10.1117/1.2398928

9. Honda N, Ishii K, Kajjmoto Y, Awazu K. Determination of optical properties of human brain tumor tissues from 350 to $1000 \mathrm{~nm}$ to investigate the cause of false negatives in fluorescence-guided resection with 5-aminolevulinic acid. J Biomed Opt. 2018;23(7):075006. https://doi.org/10.1117/1.JBO.23.7.075006

10. Bevilacqua F, Piguet D, Marquet P, Gross JD, Tromberg BJ, Depeursinge C. In vivo local determination of tissue optical properties: applications to human brain. Appl Opt. 1999;38:4939-50. https://doi.org/10.1364/ao.38.004939

11. Yaroslavsky AN, Schulze PC, Yaroslavsky IV, Schober R, Ulrich F, Schwarzmaier H-J. Optical properties of selected native and coagulated human brain tissue in vitro in the visible and near Infrared spectral range. Phys Med Biol. 2002;47:2059-2073. https://doi.org/10.1088/0031-9155/47/12/305

12. Gebhart SC, Lin WC, Mahadevan-Jansen A. In vitro determination of normal and neoplastic human brain tissue optical properties using inverse adding-doubling. Phys Med Biol. 2006;51:2011-2027. https://doi.org/10.1088/0031-9155/51/8/004

13. Ozer K, Bozkulak O, Tabakoglu HO, Kurt A, Gulsoy M. Optical properties of native and coagulated lamb brain tissues in vitro in the visible and near-infrared spectral range. In: Jacques S, Roach WP, eds. Optical Interactions with Tissue and Cells XVII. Vol 6084. SPIE;2006:60840P-1-8. https://doi.org/10.1117/12.646077

14. Azimipour M, Baumgartner R, Liu Y, Jacques SL, Eliceiri K, Pashaie R. Extraction of optical properties and prediction of light distribution in rat brain tissue. J Biomed Opt. 2014;19(7):075001-11. https://doi.org/10.1117/1.JBO.19.7.075001

15. Wood MFG, Vurgun N, Wallenburg MA, Vitkin IA. Effects of formalin fixation on tissue optical polarization properties. Phys Med Biol. 2011;56(8):115-122. https://doi.org/10.1088/0031-9155/56/8/N01

16. Aung H, De Angelo B, Soldano J, Kostyk P, Rodriguez B, Xu M. On alterations in the refractive index and scattering properties of biological tissue caused by histological processing. In: Wax AP, Beckman V, eds. Biomedical Applications of Light Scattering VII. Vol 8592. SPIE;2013:85920X-1-8. https://doi.org/10.1117/12.2005927

17. Abe M, Takahashi M, Horiuchi K, Nagano A. The changes in crosslink contents in tissue after formalin fixation. Anal Biochem. 2003;318(1):118-123. https://doi.org/10.1016/S0003-2697(03)00194-5

18. Hsiung P-L, Nambiar P, Fujimoto J. Effect of tissue preservation on imaging using ultrahigh resolution optical coherence tomography. J Biomed Opt. 2005;10(6):064033. https://doi.org/10.1117/1.2147155

19. Pitzschke A, Lovisa B, Seydoux O. et al. Optical properties of rabbit brain in the red and near-infrared: changes observed under in vivo, postmortem, frozen and formalin-fixated conditions. J Biomed Opt. 2015:20(2):025006. https://doi.org/10.1117/1.JBO.20.2.025006

20. Anand S, Cicchi R, Martelli F, et al. Effects of formalin fixation on tissue optical properties of in-vitro brain samples. In: Jansen D, ed. Optical Interactions with Tissue and Cells XXVI. Vol 9321. SPIE;2015:93210Z1-5. https://doi.org/10.1117/12.2076961 
21. Wilson BC, Patterson MS, Flock ST. Indirect versus direct techniques for the measurement of the optical properties of tissues. J Photochem Photobiol. 1987;46(55):601-608. https://doi.org/10.1111/j.1751-1097.1987.tb04820.x

22. van der Zee P. Measurement and Modelling of the Optical Properties of Human Tissue in the Near Infrared. Ph.D. Dissertation, University of London, London, U.K., 1992.

23. Prahl, S. Light Transport in Tissue. Ph.D. Dissertation, University Texas, Austin, U.S.A., 1988.

24. Roysten D, Poston R, Prahl S. Optical properties of scattering and absorbing materials used in the development of optical phantoms at 1064nm. J Biomed Opt. 1996;1(1):110-116. https://doi.org/10.1117/12.227698

25. Shahin A, Bachir W, Sayem El-Daher M. Polystyrene microsphere optical properties by Kubelka-Munk and diffusion approximation with a single integrating sphere system: a comparative study. J Spec. 2019:3406319. https://doi.org/10.1155/2019/3406319

26. Prahl, S. Inverse Adding-Doubling XP version-3-9-5; School of Medicine, Oregon Health and Science University: Portland, 2018.

27. van de Hulst HC. Light Scattering by Small Particles. New York: Dover publication; 1981.

28. Ashoor HE, Jasim Kh E. Determining the optical properties of blood using He-Ne laser and double integrating sphere set-up. Polish J Med Phys Eng. 2019;25(1):1-5. https://doi.org/10.2478/pjmpe-2019-0001

29. Friebel M, Roggan A, Muller G, Meinke M. Determination of optical properties of human blood in the spectral range 250 to $1100 \mathrm{~nm}$ using Monte Carlo simulations with hematocrit-dependent effective scattering phase functions. J Biomed Opt. 2006;11(3):034021. https://doi.org/10.1117/1.2203659

30. Lovell AT, Hebden JC, Goldstone LC, Cope M. Determination of the transport scattering coefficient of red blood cells. In: Chance B, Alfano RR, Tromberg BJ, eds. Optical Tomography and Spectroscopy of Tissue III. Vol 3597. SPIE;1999:175-182. https://doi.org/10.1117/12.356795

31. Sun Y, Fischer BM, Pickwell-MacPherson E. Effects of formalin fixing on the terahertz properties of biological tissues. J Biomed Opt. 2009;14(6):064017-1-7. https://doi.org/10.1117/1.3268439 